\title{
Considerations on the Unification of Quantum Physics with the General Theory of Relativity
}

\author{
Guido Zbiral \\ Klosterneuburg, Austria \\ Email: guido@zbiral.at \\ Received 25 April 2016; accepted 17 June 2016; published 21 June 2016 \\ Copyright (C) 2016 by author and Scientific Research Publishing Inc. \\ This work is licensed under the Creative Commons Attribution International License (CC BY). \\ http://creativecommons.org/licenses/by/4.0/ \\ (c) (i) Open Access
}

\section{Abstract}

From a holistic perspective of a physical space of any given size ${ }^{1}$, it is invariably necessary to consider its energy content, since no physical means exists of making a physical space completely devoid of energy. Such a space would therefore only be a fictive "geometric space"-that can be intellectually conceived and treated according to the rules of the appropriate geometry-although not existing in reality in the cosmos. Cosmic space always contains energy in one form or another, limited by the space under consideration. Therefore, each space possesses an energy density-no matter how low, which never becomes zero. Because of the mass-energy equivalence relationship $E \equiv m . c^{2}$, cosmic space also possesses a mass equivalent and is therefore "materialistic" in nature. If this is considered in association with Einstein's space-time, what is obtained instead is an "energytime", i.e. an energy effect, which is based on Planck's action quantum $h$. Under this condition, a close relationship would appear to exist between the General Theory of Relativity and Quantum Physics. Furthermore, it will be shown that the physical conditions of space are such that a natural quantisation of space and time exists, thus obviating the need for any artificial or arbitrary quantisation.

\section{Keywords}

Spacetime, Energy Effect, Planck's Action Quantum h, Adapted Spacetime, Quantisation of Space and Time

\section{Introduction}

\section{The Energy $\leftrightarrow$ Space-Dualism}

Due to the equivalence of mass and energy, energy can only ever appear physically—i.e. in a three-dimensional ${ }^{1}$ Each part of cosmic space is itself a real physical space. 
form. The existence (or presence) of any given state and any given "quantum" of energy thus indispensably requires the existence of a three-dimensional space in order to be able to create an "energy effect".

Axiom: everything that exists in the cosmos is energy in one or other of its diverse manifestations and nothing exists that does not contain energy.

Cosmic space can only exist in association with energy, since cosmic space itself has been created in the course of world history by the process of dispersion of energy. For example, the energy density of the almost perfectly isotropic ${ }^{2}$ cosmic background radiation (photon radiation) in space-a relic of the Big Bang, now having a temperature of approx. $2.7 \mathrm{~K}$-is currently ca. $6 \times 10^{-20} \mathrm{~J} / \mathrm{cm}^{3}$ or $0.38 \mathrm{eV} / \mathrm{cm}^{3}$.

It is the nature of cosmic space to invariably be completely filled or pervaded (penetrated) with energy. As energy only manifests itself in a quantised (discrete) form, it follows that cosmic space-as the "carrier" of energy quanta—must also be structured in a quantum manner.

The properties (or parameters) of a given space under consideration are complexly related (in accordance with physical laws) to the energy situation of this space. For this reason, energy can only ever be simultaneously considered with the physical space claimed by it. This physical space is a part of the entire cosmic space invariably. Energy without space and space without energy do not exist anywhere in the entire cosmos! From this, it follows that:

1) Energy requires space in order to yield an energy effect after a specific time (as a consequence of its inertia).

2) Space requires energy in order to be a physical space (a completely empty space void of energy would "invalidate" the concept of space) $)^{3}$.

3) Energy and space are mutually dependent and are mutually inseparably associated from a physical perspective; they should therefore always be considered "holistically". This applies equally both to the cosmos as a whole and to each single photon as a fundamental component of the entire cosmos. The frequency $v$ of the photon determines its energy $E$, while its wavelength $\lambda$ determines the space claimed by the photon. As far as the physical size is concerned [energy density $\times$ volume], energy and cosmic (physical) space are coessential or identical!

4) $E=h \times v$ (Planck's Equation), $h$ (Planck's action quantum) $=6.626 \times 10^{-34} \mathrm{~J} \cdot \mathrm{s}, v=c / \lambda[1]$.

Due to the fundamental importance of the theses 1) to 3) for the following considerations, I shall attempteven at the risk of repeating myself-to formulate even more precisely as well as justify this:

Space, considered holistically, contains energy—always and without exception.

Energy, considered holistically, claims a specific and hence limited space-always and without exception.

Note: At the very beginning, only energy existed in the highest concentrated form! As a result of the ensuing "energy dispersion", the constantly expanding cosmic space came into being and started to expand (associated by the simultaneous decrease in the energy density). Cosmic space is completely flooded by energy-always and everywhere-and is thus identical to energy. Cosmic space can therefore be considered as "inflated energy".

Cosmic space consists solely of energy in all possible existing states and energy densities. Therefore, space and energy, considered holistically from a physical perspective, are one and the same!

However, we have become accustomed in our daily life to consider the two identical physical quantities as separate entities (essences), because in one case the subject of our interest is only the volume of the space (or its geometrical form) — while in the other case it is only the amount (or density) of the energy within the space!

"Pure" space, i.e. an absolute vacuum, devoid of any energy content, is not a physical space, and thus has no physical dimension - it is only a "geometrical space" (i.e., a graphic space that can be imagined intellectually and analysed from a geometrical and mathematical perspective). Albeit possessing the geometrical spatial dimensions $\left[\mathrm{R}^{3}\right]$, say, $\left[\mathrm{cm}^{3}\right]$, it is not real in the sense of existing in the cosmos.

The complete energy vacuum of a space cannot be represented by any physical state; neither has such a space ever existed anywhere in the cosmos nor is it possible to create one- even with the most extreme technical efforts imaginable. According to quantum theory, it is not even possible to produce an ideal vacuum in the cosmos, since a field energy will never become precisely zero, its field quanta only fluctuate in an indeterminate manner around the zero value. It is these quantum fluctuations that therefore always result in a certain residual energy. This means that a supposedly "completely empty space-devoid of energy" can never exist in the cosmos, but

\footnotetext{
${ }^{2}$ No preference exists with respect to position and direction of radiation, i.e., it is uniform in all places and directions.

${ }^{3}$ According to Leibnitz: "Space is the relationship of objects to each other; space is nothing without objects present in the space." Each form of energy (三mass) shall be physically assigned the term “object”. Therefore cosmic space is “material”.
} 
must contain a certain residual field energy, no matter how small. For this reason, no heat accumulator can exist, from which it is possible to completely extract its heat content. It will always retain a residual quantity of heatno matter how small.

"Real = physical space”, i.e. the entire universe as well as any arbitrarily small part of it, always possesses an energy density $\varepsilon=\left[E / R^{3}\right]$, its “physical dimension" therefore being $\left[R^{3} \cdot \varepsilon\right]=$ energy $=[E]$, e.g. W·s or $\mathrm{J}$ or $\mathrm{N} \cdot \mathrm{m}$. Thus, for example, the energy density of the universe due to the cosmic background radiation is given by $\varepsilon=6 \times$ $10^{-20} \mathrm{~J} / \mathrm{cm}^{3}$ or $0.38 \mathrm{eV} / \mathrm{cm}^{3}$.

From this, it follows that:

Cosmic space is energy and for this reason must be classified as "material" in nature, since the entire cosmos has emerged exclusively from energy (three-dimensional) in the course of world time t, and it is at all times another energy effect, i.e. the product of energy multiplied by time.

Even by applying the greatest possible technical effort, it is physically impossible to completely separate the "energy of space" from its particular space under consideration ${ }^{4}$. Every arbitrarily small or large space remains therefore inseparably associated with its intrinsic residual energy. Exactly as is the case with energy, no part of real (physical) space-no matter how small, created by the dissipation of energy, can be annihilated; it remains in existence forever (in the expansion phase of the cosmos).

Therefore in all physical considerations, the particular space under consideration must be regarded together with its energy content, which is present at all times without exception. Considering only the geometrical dimensions of space $\left[R^{3}\right]$, may result in a physically false picture, because:

It is energy that makes space into a real physical space and provides space with both its internal structure and external form by means of the gravitation inseparably associated with energy!

In other words: each arbitrary quantum of energy is inextricably associated with space and gravitation, or alternatively, each physical space contains energy and gravitation.

\section{Association between Spacetime and Energy Effect}

1) In Einstein's spacetime, space contains no energy (and therefore no gravitational force) —it is a continuum.

Spacetime thus possesses only the geometrical dimension $R^{3}$ multiplied by time $t$ and-in the sense of the axiom set forth in Chapter 1—cannot be ascribed any real existence. It is merely a mathematical-geometrical construct, in which gravitation is interpreted not as a force but as the geometry of space.

Notwithstanding being an extremely successful theory of gravitation, all attempts to combine the General Theory of Relativity with Quantum Physics into one single united theory of quantum gravitation have hitherto failed. The unification of the two great theories of the 20th century is regarded as one of the great challenges of physics, and yet it is not certain whether this will be successful based on the premises currently existing. The requirements for the unification of the two theories would, of necessity, include the quantisation of time and space.

As a continuum, the completely empty geometrical space is only divisible to an arbitrary fine extent from a geometrical perspective-but not, however, from a physical perspective. A space completely devoid of energy, e.g. spacetime, is thus not physically quantisable, but only geometrically quantisable-by being assigned arbitrary (fictitious) space quanta, e.g. in units of the Planck length $\left(L_{P}\right)$ or a multiple thereof. In this manner, however, it is not possible to bring about a unification of these two great theories. For this purpose, it will be necessary to seek a different path.

2) If the insights obtained from Chapter 1 are included in the following physical considerations-i.e. that a “cosmic space $\equiv$ energy" equivalence exists-then it becomes possible to interpret the expression for cosmic space

"spacetime $=$ space $\times$ time" as "space $\times$ energy density $\times$ time $=$ energy $\times$ time",

possessing the physical dimension $\left[\mathrm{R}^{3} \cdot \varepsilon t\right]=[E \cdot t]$, i.e. an “energy effect”. Under this condition, Planck's energy effect (quantum theory) and Einstein's adapted spacetime (adapted General Relativity Theory) can be unified. As it is an incontestable fact that the entire cosmic space with its energy content represents an energy effect in the course of the world time to date, then the above interpretation must be permissible. The spacetime thereby "adapted" is therefore an "energy effect" and not a continuum. From a holistic perspective, cosmic space re-

${ }^{4}$ It corresponds to the 3rd Law of Thermodynamics: no heat accumulator exists, from which it is possible to completely extract its heat content. There will always remain a residual amount of heat-no matter how small. 
mains today what it has always been since its creation, namely energy. Expressing this more precisely:

As cosmic space (from a holistic perspective) and cosmic energy are coessential or identical, then adapted spacetime $=$ energy $\times$ time $=$ energy effect are similarly identical, as already determined in Chapter 1 , item c) above! By incorporating the always present space-energy into "spacetime", the latter is transformed into an "energy effect", based on the Planck's action quantum $\mathrm{h}=6.626 \times 10^{-34} \mathrm{~J} \cdot \mathrm{s}$. In this manner, a close relationship between the two great theories of the 20th century is discerned, which leads me to believe that a unification of these two theories ought to be possible in principle, because-adapted spacetime is quantisable!

\section{Considerations on a Quantisation of Real Physical Space}

As far as the quantisation of cosmic space is concerned, no confirmed research results from the community of physicists as yet exist [2]-[4].

As already indicated, the entire cosmic space can be interpreted as an "energy effect". For this reason, no element of space can exist—no matter how small—that is completely devoid of energy.

It would therefore be possible to interpret Planck's action quantum $\mathrm{h}$ as the smallest elementary unit of energy-effect, from which the entire cosmic space is composed.

Under this condition, the internal structure of cosmic space is essentially determined by the action quantum $\mathrm{h}$. In consequence, this would mean that cosmic space cannot be physically divided up into arbitrarily small amounts, but rather it is possible to imagine a smallest physical element of space, say, a "space quantum", which contains an energy quantum, such as a cosmic photon, whereby the dimensions of this space quantum are dependent on the energy situation present ${ }^{5}$.

Each energy quantum of cosmic space possesses its own (energy-specific) space quantum, and they are both identical—in precisely the same manner that energy and cosmic space are identical.

As the photons in cosmic space are "free (non-bounded) particles", the energy of the photons can, in principle, assume any arbitrary positive value. As the energy of photons in cosmic space is therefore continually changeable, so the energy density and the dimensions of each cosmic element of space are also continually changeable. We owe this situation to the continual growth (or constant expansion) of cosmic space (by contrast, we owe the stability of matter to the quantisation of energy of the system of particles that are in a bound state due to their interaction with each other).

The energetic state of each cosmic element of space is determined by the frequency of the photon acting in this element of space in accordance with Planck's Equation $E=h \times v, v=c / \lambda$.

As the photons on their path through cosmic space constantly generate entropy (being unable to escape the 2nd Law of Thermodynamics), they gradually lose their energy. In the case of the cosmic background radiation, the decrease of energy entails a decrease in the frequency $v$ (or an increase in the wavelength $\lambda$ ) of the cosmic photons $^{6}$. A low-energy photon therefore requires a greater spatial component, in order to be able to oscillate with the lower frequency (or greater wavelength). The photon effectively expands the space it claims against the effect of gravitation, thus creating more space for its movement.

Note: The wavelength $\lambda$ of blackbody radiation photons is inversely proportional to its absolute temperature $T$ and, at $T=1 \mathrm{~K}$, is approx. $2.9 \mathrm{~mm}$. The typical wavelength $\lambda$ of cosmic photons associated with the current cosmic background radiation for $T=2.7 \mathrm{~K}$ is approx. $1 \mathrm{~mm}$, corresponding to a frequency $v$ of approx. $0.3 \times$ $10^{12} \mathrm{~Hz}$.

In contrast to the space quanta created by the photons associated with the cosmic background radiation, the highest-energy photons in the history of the cosmos-and thus smallest possible space quanta-occurred immediately following the Big Bang, once the physically unimaginable, immeasurably high "initial temperature" of the cosmos at time $t=0$ had fallen to approx. $10^{32} \mathrm{~K}$. This temperature is known as Planck's temperature $T_{P}$.

With the wavelength $\lambda$ of photons at $T=1 \mathrm{~K}$ being approx. $2.9 \times 10^{-3} \mathrm{~m}$, the wavelength $\lambda_{P}$ at $T_{P}=10^{32} \mathrm{~K}$ can be approximately calculated as $10^{-3} / 10^{32}=10^{-35} \mathrm{~m}$, this being known as the Planck length $\lambda_{P}$ and-as the physically smallest possible length—also defines the dimensions of the smallest (and highest-energy) space quanta (in physics books, the Planck length is typically denoted by $L_{P}$ ).

\footnotetext{
${ }^{5}$ The smallest space quanta are therefore those currently occupied by the highest-energy photons. No space quanta smaller in scale may exist. Notwithstanding this, if the intellectual attempt is made to divide such a space quantum, then 2 space quanta would be obtained—each with a photon of half the wavelength, i.e. with twice the energy. This however is physically impossible. Therefore the smallest space quanta do indeed exist and cannot be further divided from a physical perspective.

${ }^{6}$ The typical wavelength of cavity photon radiation $\lambda$ also corresponds to the mean distance between the photons.
} 
Given the speed of light $c=3 \times 10^{8} \mathrm{~m} \cdot \mathrm{s}^{-1}$, the frequency $v_{P}=c / \lambda_{P}$ can be approximately calculated at $10^{8}$ $\mathrm{m} \cdot \mathrm{s}^{-1} / 10^{-35} \mathrm{~m}=10^{43} \mathrm{~s}^{-1}$ (or Hz,) and is known as the Planck frequency $v_{p}$.

To travel the length of $\lambda_{P}$, a ray of light requires the time $t_{P}=\lambda_{P} / c=10^{-35} \mathrm{~m} / 3 \times 10^{8} \mathrm{~m} \cdot \mathrm{s}^{-1}=10^{-43} \mathrm{~s}$, this time being known as the Planck time $t_{P}$ (the shortest interval of time physically possible).

The Planck values calculated here on a scale of orders of magnitude only represent the first-possible physical parameters in the history of the cosmos immediately following the Big Bang at $T_{P}=10^{32} \mathrm{~K}$, derived from the first (and thus most energy-rich) photons of cosmic radiation energy, which should therefore be referred to as Planck photons. These physical parameters change in the course of world time, i.e., the temperature, frequency, energy of cosmic photons is steadily decreasing, while the wavelength is steadily increasing. For this reason, the "physical quantisation of cosmic space" cannot be based on the constant Planck length $\lambda_{P}^{3}=\left(10^{-35} \mathrm{~m}\right)^{3}$-instead it is necessary to refer to the current energy situation of the cosmic photons.

Since the temperature and thus the energy of cosmic photons decrease everywhere uniformly with the falling thermal state of equilibrium of the cosmic background radiation-this corresponding to a universal uniform increase in entropy taking place, more space is required uniformly everywhere for this entropy to be deposited. This space is thereby created by the universal uniform increase in the wavelength $\lambda$ of all cosmic photons. This means that the growth rate of cosmic space is everywhere the same- the universe is expanding uniformly and isotropically. An unequivocal correlation thus emerges between Planck's formula for the energy of the cosmic background radiation and the universal uniform growth (expansion) of cosmic space. This energy-space behaviour exhibited by all cosmic photons (or space quanta) precisely corresponds to Hubble's Law ${ }^{7}$, which thus finds a physical explanation that is not derivable from the Big Bang Theory [5].

Furthermore, each quantum of space evidently possesses a three-dimensional structure and is spatially limited by the wavelength $\lambda^{3}$ of its photon. For this reason, the entire energy-filled cosmic space (as the sum total of all space quanta) must possess the same properties as its smallest energy-filled components. Everything we know about the cosmic photons and the Planck action quantum h must also apply to the cosmos as a whole. The world of the infinitesimal thus has a direct effect on the cosmos in its entirety!

Summary of the key statements contained in Chapter 3:

No space quantum exists in the cosmos without an energy quantum-were this not the case, this space quantum would be devoid of energy, and this is not possible in a physical situation. The entire cosmic space is therefore composed of energy quanta (e.g. cosmic photons), where each energy quantum in cosmic space possesses its own (energy-specific) space quantum. In the course of world time, each cosmic photon (like everything else) constantly generates entropy, the deposition of which constantly requires more cosmic space. This increase in entropy causes the photon to constantly lose energy, this being expressed in a constant increase in its wavelength $\lambda$. The increase in $\lambda$ is tantamount to a uniform expansion in the space required by each cosmic photon in all directions in order to be able to oscillate with the ever greater wavelength $\lambda$. As a result of this quantum phenomenon, all cosmic photons taken together are the cause of the constant and isotropic expansion of the entire cosmic space, precisely corresponding to Hubble’s Law.

\section{Considerations on a Quantisation of Time}

The quantisation of time is an area of research still in its infancy, concerning which there are at the present time no as yet generally recognised or confirmed results from the community of physicists available [6]. Since in General Relativity, space and time are interlocked in the spacetime continuum, if one of them is quantised, then so must the other.

To use the shortest possible constant Planck time $\left(t_{P}=10^{-43}\right.$ secs) for the quantisation of time would not be physically justifiable, but merely an arbitrary imposition. The time quanta of cosmic space will not have remained constant in the course of world time to date, but rather-in analogy to the dimensions of space quanta depending on the variable energy of the cosmic photon radiation - are dependent on the variable energetic ambient conditions of the space in question. I therefore propose to draw on the frequency $v=1 / t$ of cosmic photons for the physical quantisation of time, since Planck's Equation $E=h \times v$ also provides a correlation between space quanta and time quanta.

At the present time, the typical wavelength $\lambda$ of the cosmic photons of the cosmic background radiation is

\footnotetext{
${ }^{7}$ The same situation arises everywhere-irrespective of the point of view of the observer, i.e. an increase in the speed of expansion in propor-
} tion to the distance from the respective observer. 
approx. $1 \mathrm{~mm}$ at a cosmic temperature $T=2.7 \mathrm{~K}$, this corresponding to a frequency $v$ of $0.3 \times 10^{12} \mathrm{~Hz}$ and $t$ (at $T=2.7 \mathrm{~K})=$ ca. $3 \times 10^{-12} \mathrm{~s}$.

For $\lambda\left(\right.$ at $\left.T=273 \mathrm{~K}=0^{\circ} \mathrm{C}\right)=10^{-2} \mathrm{~mm}, v=0.3 \times 10^{14} \mathrm{~Hz}, t($ at $273 \mathrm{~K})=3 \times 10^{-14} \mathrm{~s}$

For $\lambda($ at $T=3000 \mathrm{~K})=0.9 \times 10^{-3} \mathrm{~mm}, v=0.3 \times 10^{15} \mathrm{~Hz}, t($ at $3000 \mathrm{~K})=3 \times 10^{-15} \mathrm{~s} \ldots$ and so on.

For $\lambda_{P}\left(\right.$ at $\left.T=10^{32} \mathrm{~K}\right)=10^{-35} \mathrm{~m}, v_{P}=10^{43} \mathrm{~Hz}, t_{P}\left(\right.$ at $\left.10^{32} \mathrm{~K}\right)=10^{-43} \mathrm{~s}$.

Under these considerations, the time quanta t of cosmic space is variable within the above threshold values of frequency and temperature of cosmic photons (cosmic background radiation).

Remark on spacetime: space and time, as understood by Einstein, are not independent entities but interwoven in spacetime, i.e., a physical dependence exists between them. From this it thus follows: if cosmic space is quantifiable as a perpetual carrier of energy, then cosmic time must also be quantifiable, as is seen above. Furthermore, a correlation must exist between space quanta and time quanta. Both apply in Chapter 3 and 4 . However, no evidence of spacetime quantisation currently exists, and in General Relativity, space and time are continuous and not quantisable.

\section{Conclusions}

This paper depicts how it is possible to undertake a quantisation of space based on physical arguments, whereby each space quantum is fully occupied by an energy quantum (e.g. cosmic background radiation photon). Space quanta and energy quanta are identical in the same manner that cosmic space and energy are.

The quantisation of time is derived from the frequency of the energy quanta and is thus physically associated with the space quanta. The quantisation of space and time is an absolute prerequisite to achieve a unification of Quantum Physics with the General Theory of Relativity.

\section{Acknowledgements}

My warmest thanks go to my translator C. A. Szwaja (M.A. Physics, Oxon), both for translating my manuscript from German into English and his numerous valuable suggestions on the text itself.

\section{References}

[1] Barrow, J.D. (2002) The Constants of Nature; From Alpha to Omega Wikipedia_Planck’s Constant: https://en.wikipedia.org/wiki/Planck constant

[2] Odenwald, S. (2011) The Astronomy Cafe-Ask the Astronomers: Is Space Really Quantized? www.astronomycafe.net/qadir/BackTo286.html

[3] (2013) Physics Forums: Is Space Itself Quantized? https://www.physicsforums.com/threads/is-space-itself-quantized.707694/

[4] (2011) Physics Forums: Are Space and Time Really Quantized? https://www.physicsforums.com/threads/are-space-and-time-really-quantized.509659/

[5] Zbiral, G. (2013) International Journal of Theoretical and Applied Physics, 3, 25-35.

[6] Ben Crowell, June 13, 2013 in Physics Stack Exchange: Is Time Quantized? Is There a Fundamental Time Unit That Cannot Be Divided? http://physics.stackexchange.com/questions/67899/is-time-quantized-is-there-a-fundamental-time-unit-that-cannot-bedivided 


\section{Submit or recommend next manuscript to SCIRP and we will provide best service for you:}

Accepting pre-submission inquiries through Email, Facebook, Linkedin, Twitter, etc A wide selection of journals (inclusive of 9 subjects, more than 200 journals)

Providing a 24-hour high-quality service

User-friendly online submission system

Fair and swift peer-review system

Efficient typesetting and proofreading procedure

Display of the result of downloads and visits, as well as the number of cited articles

Maximum dissemination of your research work

Submit your manuscript at: http://papersubmission.scirp.org/ 